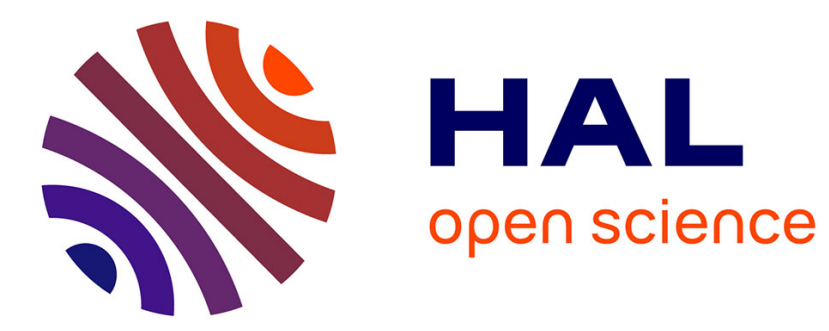

\title{
Scanning electron microscope image signal-to-noise ratio monitoring for micro-nanomanipulation.
}

\author{
Naresh Marturi, Sounkalo Dembélé, Nadine Piat
}

\section{To cite this version:}

Naresh Marturi, Sounkalo Dembélé, Nadine Piat. Scanning electron microscope image signal-to-noise ratio monitoring for micro-nanomanipulation.. The Journal of Scanning Microscopies., 2014, pp.1-11. 10.1002/sca.21137 . hal-01051309

\section{HAL Id: hal-01051309 https://hal.science/hal-01051309}

Submitted on 25 Jul 2014

HAL is a multi-disciplinary open access archive for the deposit and dissemination of scientific research documents, whether they are published or not. The documents may come from teaching and research institutions in France or abroad, or from public or private research centers.
L'archive ouverte pluridisciplinaire HAL, est destinée au dépôt et à la diffusion de documents scientifiques de niveau recherche, publiés ou non, émanant des établissements d'enseignement et de recherche français ou étrangers, des laboratoires publics ou privés. 


\title{
Scanning Electron Microscope Image Signal-to-Noise Ratio Monitoring for Micro-Nanomanipulation
}

Naresh Marturi (corresponding author), Sounkalo Dembélé and Nadine Piat

\author{
Automatic control and Micro Mechatronic Systems (AS2M), Institute FEMTO-ST, \\ Université de Franche-Comté/CNRS/ENSMM/UTBM, 24 rue Savary, 25000 Besançon, France \\ Email: naresh.marturi@femto-st.fr
}

This work is conducted with a financial support from the project NANOROBUST (ANR-11-NANO-006) funded by the Agence Nationale de la Recherche (ANR), France. It is also performed in the framework of the Labex ACTION (ANR-11LABX-01-01) and the Equipex ROBOTEX (ANR-10-EQPX-44-01) projects.

Key words: Scanning electron microscope, signal-to-noise ratio, image quality estimation, performance evaluation, micronanomanipulation.

\begin{abstract}
As an imaging system, scanning electron microscope (SEM) performs an important role in autonomous micro-nanomanipulation applications. When it comes to the sub micrometer range and at high scanning speeds, the images produced by the SEM are noisy and need to be evaluated or corrected beforehand. In this article, the quality of images produced by a tungsten gun SEM has been evaluated by quantifying the level of image signal-to-noise ratio (SNR). In order to determine the SNR, an efficient and online monitoring method is developed based on the nonlinear filtering using a single image. Using this method, the quality of images produced by a tungsten gun SEM is monitored at different experimental conditions. The derived results demonstrate the developed method's efficiency in SNR quantification and illustrate the imaging quality evolution in SEM.
\end{abstract}

\section{Introduction}

Vision-based control, also known as visual servoing, refers to the use of image information in order to control the motion of a robotic device. This technique uses the images acquired from a vision sensor to extract the information reflecting relative spatial position between the camera and the robot (Hutchinson et al., 1996; Chaumette and Hutchinson, 2006). In the recent years, it has become very popular especially in the field of robotic manipulation due to its ability in producing accurate and robust control. This has encouraged many researchers to extend this technique to micro-nanoscale in order to solve various problems associated with micro-nanomanipulation. Basically at this small scale $(<10 \mu \mathrm{m})$, manipulations are often performed using a scanning electron microscope (SEM), a transmission electron microscope (TEM) or an atomic force microscope (AFM). With its ability of producing images at high magnification in real-time, a SEM is always favored as an imaging tool for automatic micro-nanomanipulation applications.

In general, visual servoing control strategies are velocity-based and require a minimum frame rate of about 20 Hz. This corresponds to a very high scan rate compared to the normal operation of SEM. Moreover, to perform a vision-based micromanipulation task using SEM, one main requirement is that the captured images are to be noiseless and the details present in the images are high enough to be exploitative. Unfortunately, the noise level is critical in a SEM, especially at high acquisition rates. Each step during the image formation process adds noise which arise the difficulty in understanding and handling the final image noise. Practically, noise variation can be determined by acquiring images with varying scan time per pixel. If this time increases, the level of signal increases as the beam will spend more time at the particular location. However, with an increase in the scan time, the time to capture one frame increases which is not an acceptable constraint for high speed visual servoing applications. So, in order to perform a visual servoing-based micromanipulation task, it is mainly required to monitor the acquired final image quality until the overall task is accomplished. This monitoring process has been given the major attention in this paper.

In order to estimate the image quality, the primary requirement is to identify the type of final image noise. Reimer (1998) provided a detailed description of SEM and pointed out the fact that the noise added due to the primary electron emission is shot noise and grow in proportion to the useful signal. It is added due to the fluctuations in the count of emitted primary beam electrons. This type of noise follows Poisson distribution. For SEMs with thermionic guns, this shot noise is more (Sim et al., 2004b). Timischl et al., (2012) have described five sources of noise in a SEM: primary emission, secondary emission, scintillator, photocathode, and photomultiplier. They have showed every noise correspond to quantum fluctuations and can be modeled by 
Poisson statistics. However, many works assume that the noise added by the detection system is negligible (Sim et al., 2004b; Goldstein et al., 2003). Mulapudi and Joy (2003) have showed empirically that in a thermionic gun SEM, final image noise follows Gaussian statistics but not Poisson statistics. In order to generate virtual SEM images, Cizmar et al. (2008) have considered that the final image noise is an addition of a Poisson distribution representing primary emission and a Gaussian distribution representing the others types of noise in the SEM (secondary emission and electronics). Sim et al., (2004a; 2011; 2012) have studied the final noise in SEM images. They assumed this noise is additive white noise and have developed many methods for estimating image signal-to-noise ratio (SNR) based on image cross-correlation. Since these previous investigations create ambiguity in selecting the type of noise, in this work, we first study the final image noise.

To assess the quality of acquired frames, image SNR has been selected as an evaluation parameter in this work. SNR is a commonly used term in the field of signal processing to differentiate or to estimate the level of noise present in a recorded signal (Bose et al., 2004). In general terms, it is defined as the ratio of desired signal $f$ over background noise $n$ and is given by Equation (1).

$$
S N R=10 \log _{10}\left(\frac{\text { variance }(f)}{\text { variance }(n)}\right) \quad[d B]
$$

So far in the literature, many research works have proposed various methods using two SEM micrographs for the computation of SNR using auto-correlation technique (Frank and Al-Ali, 1975; Erasmus, 1982). The main drawback associated with these methods is that the acquired images are to be perfectly aligned and are obtained by scanning the same specimen area. In addition, they require long processing time and are complex to integrate and use with real time systems.

This work has been performed to monitor the image quality during an automatic vision-based micronanomanipulation task. In this paper, we present a simple and fast SNR estimation method using a single image. An estimate of the image noise to use with the developed method has been computed using non-linear spatial filtering. As the overall processing time is less, the developed method can be used with real time applications. Using the developed method, the image SNR of a tungsten filament gun SEM has been monitored during various experimental conditions that includes varying scan times, magnifications, operating time and device focus as well as during an automatic nanopositioning task.

\section{Materials and Methods}

\section{Experimental set-up}

The main set-up used for the experiments is shown in Figure 1. It consists of a JEOL JSM 820 SEM equipped with tungsten filament gun, a conventional Everhart-Thornley SE detector, an image acquisition system (DISS5 from point electronic GmbH), a SEM control computer (Intel Pentium 4, CPU $2.24 \mathrm{GHz}$ and $512 \mathrm{MB}$ of RAM) and a work computer (Intel Core 2 Duo, CPU $3.16 \mathrm{GHz}$, and $3.25 \mathrm{~GB}$ of RAM). The accelerating voltage for the SEM varies from $0.3 \mathrm{kV}$ to $30 \mathrm{kV}$ and the magnification varies from 10x to 100,000x. The maximum allowable electronic working distance is $50 \mathrm{~mm}$. The communication between the two computers is accomplished using a client-server model. The server program runs from the SEM control computer that is interfaced with SEM control electronics and the image acquisition system. The image acquisition system is mainly responsible for sending the scan parameters to SEM and to acquire the data coming from secondary electron detector. Later this data is amplified, digitized by a $4 \times 12$ bit A/D converter (DISS5) and saved as an image in the server computer. The acquired image is then transferred to the client over TCP/IP where the process of monitoring takes place. The SEM chamber is also equipped with three degrees of freedom (xyz) piezoelectric positioning platform (TRITOR 100 from Piezosystem Jena $\mathrm{GmbH}$ ). It can provide a motion up to $100 \mu \mathrm{m}$ on all the three axes with a resolution of $0.2 \mathrm{~nm}$ in open loop. Different axes of this piezo positioning platform are controlled by the NV $40 / 3$ piezo voltage amplifier. For visual servoing tasks, the platform control voltage values are transferred to the NV 40/3 from the work computer via RS-232 (serial port).

\section{Study of final image noise}

As mentioned, noise sources are numerous in SEM imaging and each contribute independently towards the final image formation. At the first hand, there are random statistical fluctuations in the primary and secondary electron emissions which produce shot noises independent from one pixel to another and depends on the material being 
used for imaging. Generally this type of noise satisfies the Poisson statistics:

$$
f(\lambda)=\frac{\mu^{\lambda}}{\lambda !} e^{-\mu}
$$

where, $\lambda$ the number of occurrences of the events and $\mu$ is the mean signal level. However, if the mean number of events is large enough $(\mu>10)$, the Gaussian distribution is a good approximation for the Poisson distribution. On the other hand, we have also noise added by the detection and recording system. Apart from this, noise is also added by the photomultipliers where they emit some signal even in the absence of original signal. This noise is due to the thermal fluctuations in the photocathode. Upon considering all these noises, the final image noise $n$ (x, $\mathrm{y})$ at the pixel $(\mathrm{x}, \mathrm{y})$ is a result of superposition of all these partial noises. Under the central limit theorem this final noise can be approximated with Gaussian statistics (Bovik, 2010).

\section{Developed method for SNR quantification}

From the above study, we assume that the final acquired image $g(x, y)$ of silicon microparts sample shown in Figure 2(A) is formed by the addition of additive white noise $n(x, y)$ to the original noise free image $f(x, y)$.

$$
g(x, y)=f(x, y)+n(x, y)
$$

As an initial step, the contrast of the image is adjusted using histogram equalization. This is optional as most of the present day microscopes come with an integrated module to perform this task during image acquisition. Next, the median filter mask of appropriate size is selected based on the amount of blurring and the total amount of processing time taken. The amount of blurring has been computed by calculating the mean squared error (MSE) between the original image and the filtered image $\hat{f}(x, y)$ given by Equation (4) (Bose et al., 2004).

$$
M S E=\frac{1}{M N} \sum_{i=1}^{M} \sum_{j=1}^{N}(f(x, y)-\hat{f}(x, y))^{2}
$$

where, $M$ and $N$ are the image dimensions. In order to accelerate the overall process, a fast sorting algorithm is used in developing the median filter. Now, the goal is to filter $g(x, y)$ by applying the median filter to find a closest estimate $\hat{f}(x, y)$ of the noise free image $f(x, y)$. Thus

$$
\hat{f}(x, y)=\operatorname{median}\{g(s, t)\}
$$

where, $(s, t)$ are the local filter neighborhood pixel coordinates selected from the acquired image. Figure 2(B) shows the filtered image $\hat{f}(x, y)$ of Figure 2(A). In turn this filtered image is subtracted from the original image to produce a noise image $n(x, y)$ given by Equation (6). Inverted version of the noise image (black regions correspond to noise) is shown in Figure 2(C).

$$
n(x, y)=g(x, y)-\hat{f}(x, y)
$$

From the noise image, it is clear that the slight edges of microparts (can be treated as signal) are also appeared in the noise image. Since, estimating the noise using a single image is under constrained, it is difficult to estimate the accurate noise level by filtering. However, many works have pointed out this problem and proposed some solutions to tackle this issue (Tai et al. 2008). Since this work is mainly aimed at performing quick monitoring of image quality to assist the final visual servoing task, we do not consider estimating the accurate amount of noise.

Finally, using the estimated noise free image $\hat{f}(x, y)$ and noise image $n(x, y)$, the SNR is computed using Equation (7) obtained from Equation (1).

$$
S N R=20 \log _{10}\left(\frac{\sigma_{\widehat{f}(x, y)}}{\sigma_{n(x, y)}}\right) \quad[d B]
$$

where, $\sigma=\sqrt{\text { variance }}, \sigma_{\hat{f}(x, y)}$ and $\sigma_{n(x, y)}$ are the standard deviations of filtered and noise images, respectively.

\section{Experimental samples and conditions}

All the experimental codes are programmed in $\mathrm{C}++$ and are executed from the work computer. The micro- 
nanostructures used for various experiments are fabricated in the micro-manufacturing center at FEMTO-ST. The sample used for the noise study, scan time, magnification and focus experiments is a gold on silicon microscale calibration pattern (Marturi, 2013) containing multiple chess grid regions with squares ranging from $25 \mu \mathrm{m}$ down to $1 \mu \mathrm{m}$ per side. All the experiments are conducted using an aperture size of $50 \mu \mathrm{m}, 10 \mathrm{kV}$ accelerating voltage, at 1000x magnification. The magnification test is a completely automated process where the magnification values are transferred to the DISS5 device from work computer via image server. The magnification is digitally changed by varying the size of the scan length on the sample. The drift observed at high magnifications has been corrected automatically using the method presented by Marturi et al. (2013b). For focus experiment, the DISS5 image acquisition system used for this work provides a simple control for the focus by linking the objective lens focal length with a series of focus steps (i.e. each focus step modifies the focal length to get a focused image). The focus steps are varied automatically and more details can be found in (Marturi et al., 2013a). The images are acquired with a scan time of $360 \mathrm{~ns}$ for both magnification and focus experiments.

For monitoring the image quality in real-time the sample used is a silicon wafer containing the microstructures of dimensions $6 \mu \mathrm{m} \times 6 \mu \mathrm{m} \times 2.5 \mu \mathrm{m}$ (Figure 2). They were designed to be assembled by their respective U-shaped grooves (without using any external adhesives) to form three dimensional structures (Dembélé et al., 2012). These type of 3D structures can be used for building complex micro-electro-mechanical systems (MEMS). For this test, the magnification used is $5000 \mathrm{x}$, accelerating voltage is $10 \mathrm{kV}$, scan time of $360 \mathrm{~ns}$ and aperture size of $30 \mu \mathrm{m}$. The sample used with automatic positioning experiment is the silicon micropart (Figure 3) of dimensions $10 \mu \mathrm{m} \times 100 \mu \mathrm{m} \times 20 \mu \mathrm{m}$. This experiment has been conducted in order to characterize the surface stiffness of the micropart. For this test the images are acquired using an accelerating voltage of $10 \mathrm{kV}$ at $1000 \mathrm{x}$ magnification. For all the experiments, the SEM secondary electron images of dimensions 512 x 512 pixels are used.

\section{Results}

\section{Study of the final image noise}

This test has been conducted to study the final image noise with fast scan rates. It is performed by acquiring the images of calibration pattern at different scan times and by computing the noise distribution within a single region (Figure 4(A) and 4(B)). The scan times used for this test are: $180 \mathrm{~ns}$ (least possible with our system) and $360 \mathrm{~ns}$. Two plain image regions are selected for each image where one region contains only gold and the other contain only silicon. Figure $4(\mathrm{C})$ to $4(\mathrm{~F})$ show the intensity histograms along with the fitted distributions (Gaussian and Poisson) for gold and silicon regions at different scan times.

\section{Filter size selection}

The artificial noise free and noisy images (Figures 5(A) and 5(B)) of gold on carbon generated by Artimagen library (Cizmar et al. 2008) are considered for this purpose. It is a library provided by the National Institute of Standards and Technology (NIST) to produce artificial SEM images with varying noise. The additive Gaussian noise with a standard deviation equal to 0.07 has been added to generate a noisy image. The MSE between original image and noisy image is found to be 110.30. The computed MSE values along with the total processing time taken using different filter sizes are summarized in Table I. The plots shown in the Figure 6(A) to 6(D) are the intensity values of the original image and filtered image along the horizontal line shown in the Figure 5(A).

\section{Precision testing of the developed approach}

The precision of the developed method is tested by using a noise free image (Figure 5(A)) and by corrupting it with a known amount of noise (SNR). Later the SNR values of the corrupted images are computed using the proposed method and are compared with the known values. Table II summarizes the computed values along with the percent error between the filtered image and the original image.

\section{Quality monitoring with respect to scan time}

Initial tests are performed to evaluate the image quality with a change in the scan time. It is a well-known fact that the SNR increases with increase in scan time. This experiment has been performed in order to test and support this statement. Nine different images of gold on carbon sample (at 30,000x magnification) and calibration pattern (at 1000x magnification) are acquired with varying scan times. Figure 7 shows the result of monitoring process.

\section{Quality monitoring with respect to magnification}

The second tests are performed to monitor the SNR evolution with change in magnification. For this test, 
images of the calibration pattern are acquired for the magnifications ranging from 250x to 1000x with a step of 10. For each magnification one image is acquired and the SNR is computed directly at the time of acquisition. Figure 8 shows the evolution of the SNR with respect to magnification.

\section{Quality monitoring with respect to focus}

In this test, the images of the calibration pattern are acquired for a series of focus steps (change in focus) and the image SNR has been monitored. Figure 9 shows the variation of SNR along with image sharpness with change in the focus steps. The maximum of the sharpness curve provides the best focus position. For this test the image sharpness $S$ has been computed using normalized variance sharpness function given by Equation (8) (Marturi et al., 2013a).

$$
S=\frac{1}{M N} \frac{1}{\mu} \sum_{M} \sum_{N}(g(x, y)-\mu)^{2}
$$

where, $M$ and $N$ are the image dimensions, $\mu$ is the mean intensity of the image $g(x, y)$ given by Equation (9).

$$
\mu=\frac{1}{M N} \sum_{M} \sum_{N} g(x, y)
$$

\section{Quality monitoring with respect to time}

In this test, the SNR quantification and image quality monitoring is performed with instrument operational time. The total operational time selected is 45 minutes. As the overall operating time is larger, the specimen surface is connected to the mounting plate using a copper conductive tape to remove the accumulated charge on the sample surface. Total time taken to acquire, transfer and process a single image is about 400 milliseconds. The obtained SNR values during this test are plotted and shown in the Figure 10. The output data has been fitted using polynomial fitting in order to see the actual response.

\section{Quality monitoring with respect to time during nanopositioning}

The characterization of an optoelectronic microstructure is now considered. The structure is arranged horizontally on the platform, the vertically disposed probe comes into contact with the structure to estimate its stiffness. The structure must be positioned more accurately and quickly as possible under the probe. The best solution is to use the images provided by the SEM to achieve the control of the platform. But if the signal level is not high enough, so the SNR high enough, it will not be possible to measure the information necessary for accomplishing the task. Monitoring the SNR of images should allow avoiding using the images that do not have the level of SNR required. This required level will be determined after performing visual feature extraction tests at different experimental conditions and is not discussed in this paper. The experiment starts after stabilization about 40 minutes after the ignition of the SEM. Figure 11(A) and 11(B) show the reference and initial images. Figure 11(C) to 11(E) shows the error during the task and Figure 11(F) shows the final error or finish of the positioning task. The positioning error is computed and compensated by the visual servoing control strategy (Marturi, 2013). The corresponding evolution of the SNR of the images for each iteration is shown in Figure 12.

\section{Discussion}

After analyzing the intensity histograms and fitted distributions (Figure 4), it is clear that any of the two distribution curves (Poisson and Gaussian) do not fit correctly with the real pixel intensity distributions. However, Gaussian distribution seems to provide better fit than Poisson distribution which can be clearly seen with the intensity histograms especially with silicon regions.

From Table I, it can be seen that filter size of $5 \times 5$ establishes a good compromise between accuracy and time of SNR computation with the current setup. With another experimental setup, the choice may vary. Upon testing the precision of the developed method in estimating the original SNR, the mean variation of the error is comparatively less for $5 \times 5$ filter (Table 2) and the percent error is less than 10 in most of the cases (with a filter size of $5 \times 5$ ). However, with high amount of noise in the image (especially for SNR $<12 \mathrm{~dB}$ ) the percent error is more than 10. This is mainly due to the inability of median filtering in the presence of high amount of noise. In fact, a median filter is able to remove noise in the images only if the noise pixels are less than one half of its neighborhood. This is the reason why a $3 \times 3$ filter provides poor error and a $7 \times 7$ filter provides better error than a 5 x 5 filter (for some conditions). However, from the performed experiments with our system, it has been found that the SNR level is more than $14 \mathrm{~dB}$ in all cases. 
Monitoring the SNR with respect to scan time has quantified a result known qualitatively, namely that increasing the dwell time leads to an increase in the SNR of images. The obtained results make this clear i.e., the image quality increases with increase in the dwell time. This phenomenon can be explained as that the increase in dwell time reduces the overall raster rate and increases the amount of time the beam spends at a particular location. Because of this, the electron count recorded at the detector increases subsequently improving the image quality. Besides, to control a robot using visual feedback high frequency acquisition rate is required that is possible only by using a lowest dwell time. We must therefore ensure that the dwell time selected provides a sufficient level of SNR.

The evolution of the SNR with respect to magnification is a nonuniform decreasing function. With an increase in the magnification, since the size of the monitor (image) is fixed, the size of the scan area is narrowed and the surface corresponding to scan a pixel reduces. Therefore, the area of the gold region that actively participate in generating the dominant quantity of original signal reduces due to which the SNR reduces. This phenomena mostly depends on the material of the sample used for imaging.

The results obtained by monitoring the imaging quality with respect to focus show that the SNR level varies simultaneously with image sharpness. This phenomenon can be explained in two ways. The primary one is, with a change in the focus step (working distance), the size of beam interacting with the sample surface varies. At the point of in-focus, the current density is high resulting in more signal from the sample improving the SNR. The second one is, with a change in the focus, the level of original signal details present in the image varies. At the point out-of-focus, noise level is predominantly more than that of the signal level, e.g. at the initial steps of the sharpness curve, the images are completely dark containing no image details other than noise.

There are two aspects in the evolution of image SNR over time: a random aspect and a dynamic aspect. The first aspect is due to the inherently random nature of the noise as well as electronic signal. The secondary electron emission from the interaction of the primary electrons with the electrons of the atomic cloud on a sample surface is a random phenomenon. The second aspect is characterized by a rapid increase of SNR to a peak for a time of about $500 \mathrm{~s}$, followed by a rapid decay to a minimum for a time of about $750 \mathrm{~s}$, and an irregular growth up to stabilization for a time of the order of $2500 \mathrm{~s}$. This time corresponds to the time taken by the filament to reach a specific temperature to produce stable number of electrons. As a result it is recommended to wait at least 40 minutes from the ignition of JSM 820 before starting an experiment. The successful implementation of nanopositioning using image feedback (Figure 11) confirms the validity of this recommendation.

\section{Conclusion}

The SEM is by far an important tool for producing static images with high resolution. Today, the trend is to use the SEM as a vision sensor for performing robotic manipulation of microstructures. In this case the observed scene is dynamic and requires fast image acquisition. But electronic imaging is by far known for its sensitivity to the scanning frequency i.e., a high frequency of acquisition leads to low quality images. In this case, it is necessary to analyze the image quality beforehand. In this paper, we have developed a method to quantify the level of image SNR. It is based on image non-linear filtering in the spatial domain and can be used in real time for quality monitoring. Using the developed method, image quality of a tungsten gun SEM has been monitored with respect to the following parameters: scan time, magnification, focus and operational time. The main lesson that can be drawn from these experiments is the instability of the SNR: it varies randomly and greatly depending on time as well as magnification. The analysis of the average change over time revealed two phases. In the first phase, the ratio increases and decreases suddenly. It corresponds to the rise in temperature of the filament. The second phase is a slow growing of the ratio that corresponds to a temperature stability of the filament over time. The great merit of this work done allows the quantification of the instability of SEM imaging. In the future, the method will be applied to two other types of SEMs (a LaB6 gun SEM and a FEG SEM) to study the evolution of the SNR of their images. 


\section{References}

Bose T, Meyer F, Chen MQ. 2004. Digital signal and image processing. J. Wiley.

Bovik AC. 2010. Handbook of image and video processing. Elsevier.

Chaumette F, Hutchinson S. 2006. Visual servo control. I. Basic approaches. IEEE Robot Autom Mag 13(4): 8290.

Cizmar P, Vladar AE, Ming B, Postek MT. 2008. Simulated SEM images for resolution measurement. Scanning 30 (5): 381-391.

Dembélé S, Fort-Piat NL, Marturi N, Tamadazte B. 2012. Gluing free assembly of an advanced 3D structure using visual servoing. Proceedings of Micromechanics and Microsystems Europe Workshop.

Erasmus SJ. 1982. Reduction of noise in TV rate electron microscope images by digital filtering. J Microsc 127 (1): 29-37.

Frank J, Al-Ali L. 1975. Signal-to-noise ratio of electron micrographs obtained by cross correlation. Nature 256: 376-379.

Goldstein J, Newbury DE, Joy DC, Lyman CE, Echlin P, Lifshin E, Sawyer L, Michael JR. 2003. Scanning electron microscopy and X-ray microanalysis. Springer.

Hutchinson S, Hager GD, Corke PI. 1996. A tutorial on visual servo control. IEEE Trans Rob Autom 12(5): 651670.

Marturi N. 2013. Vision and visual servoing for nanomanipulation and nanocharacterization using scanning electron microscope, Ph.D. Thesis. Université de Franche-Comté, France.

Marturi N, Tamadazte B, Dembélé S, Piat N. 2013a. Visual Servoing-Based approach for efficient autofocusing in Scanning Electron Microscope. Proceedings of the IEEE/RSJ International Conference on Intelligent Robots and Systems, in press.

Marturi N, Dembélé S, Piat N. 2013b. Fast image drift compensation in scanning electron microscope using image registration. Proceedings of the IEEE International conference on automation science and engineering: 807-812.

Mulapudi S, Joy D. 2003. Is SEM noise Gaussian. Microsc Microanal 9 (S02): 982-983.

Reimer L. 1998. Scanning Electron Microscopy: Physics of Image Formation and Microanalysis. Springer.

Sim KS, Chuah HT, Cheng Z. 2004a. Robust image signal-to-noise ratio estimation using mixed lagrange time delay estimation autoregressive model. Scanning 26(6): 287-295.

Sim KS, Nia ME, Tso CP. 2013. Noise variance estimation using image noise cross-correlation model on SEM images. Scanning 35 (3): 205-212.

Sim KS, Nia ME, Tso CP. 2011. Image noise cross-correlation for signal-to-noise ratio estimation in scanning electron microscope images. Scanning 33(2): 82-93.

Sim KS, Thong JTL, Phang JCH. 2004b. Effect of shot noise and secondary emission noise in scanning electron microscope images. Scanning 26(1): 36-40.

Tai SC, Yang SM. 2008. A fast method for image noise estimation using laplacian operator and adaptive edge detection. 3rd International symposium on communications, control and signal processing: 1077-1081.

Timischl F, Date M, Nemoto S. 2012. A statistical model of signal-noise in scanning electron microscopy. Scanning 34(3): 137-144. 


\section{LIST OF FIGURES}

Figure 1: Experimental set-up.

Figure 2: Images of silicon microparts demonstrating the developed method. (A) Final acquired image (B) filtered image using a filter size of $5 \times 5$ (C) noise image.

Figure 3: Silicon microparts used for positioning experiments.

Figure 4: Images of gold on silicon calibration pattern acquired at (A) $180 \mathrm{~ns} /$ pixel (B) $360 \mathrm{~ns} /$ pixel. Intensity histograms with approximated distributions for (C) and (E) gold regions (D) and (F) silicon regions for both images respectively.

Figure 5: Artificially generated (A) noise free and (B) noisy images of gold on carbon using Artimagen library.

Figure 6: Plots of intensities along the horizontal line shown in Figure 5(A) after filtering using the filter size of (A) $3 \times 3$ (B) $5 \times 5$ (C) $7 \times 7$ (D) $9 \times 9$.

Figure 7: Evolution of SNR with respect to scan time.

Figure 8: Evolution of SNR with respect to magnification.

Figure 9: SNR and image sharpness variation with respect to focus steps.

Figure 10: Evolution of SNR with the device operational time.

Figure 11: Series of images depicting the nanopositioning task. (A) Desired position. (B) Initial image. (C) - (E) Errors at different positions. (F) Final error.

Figure 12: Evolution of SNR during nanopositioning task. Error positions shown in Figure 11 are pointed with the boxes. 


\section{LIST OF TABLES}

Table I: Computed MSE values and processing time with various filter sizes.

Table II: Known and computed SNR values using the developed method for artificial gold on carbon image with different filter sizes. 


\section{FIGURES}

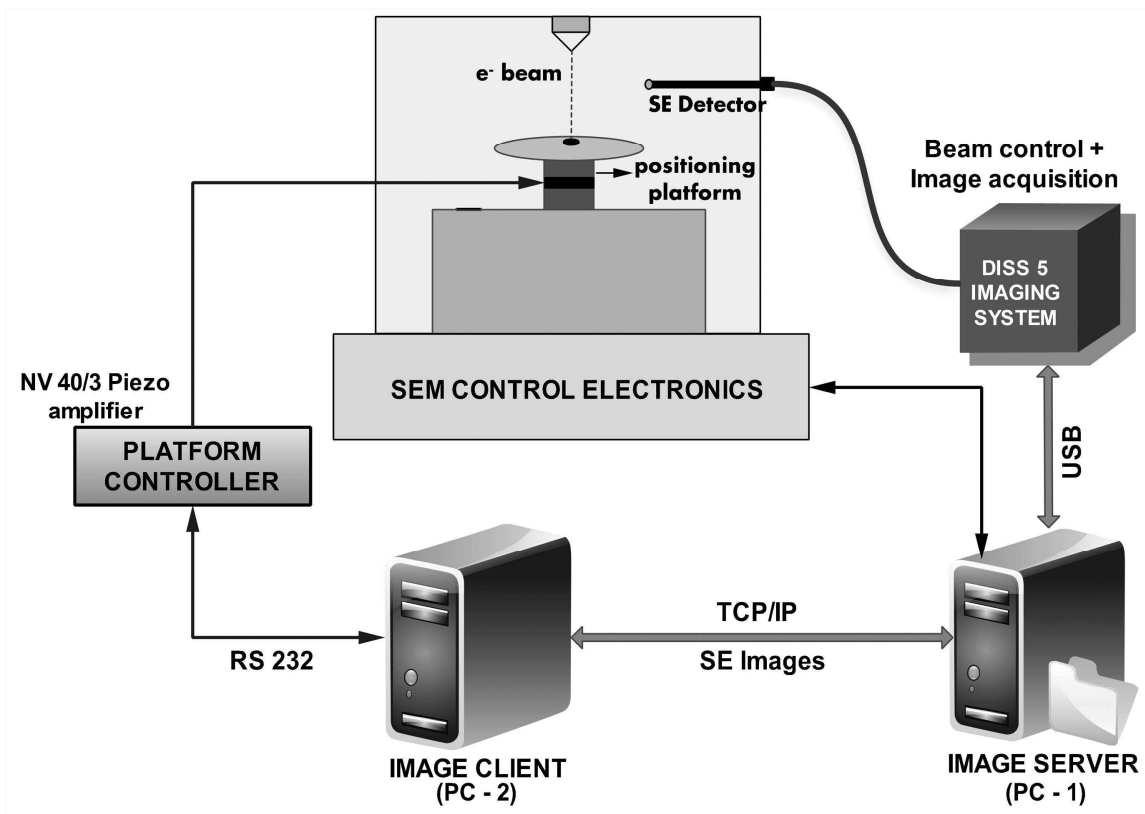

Figure 1
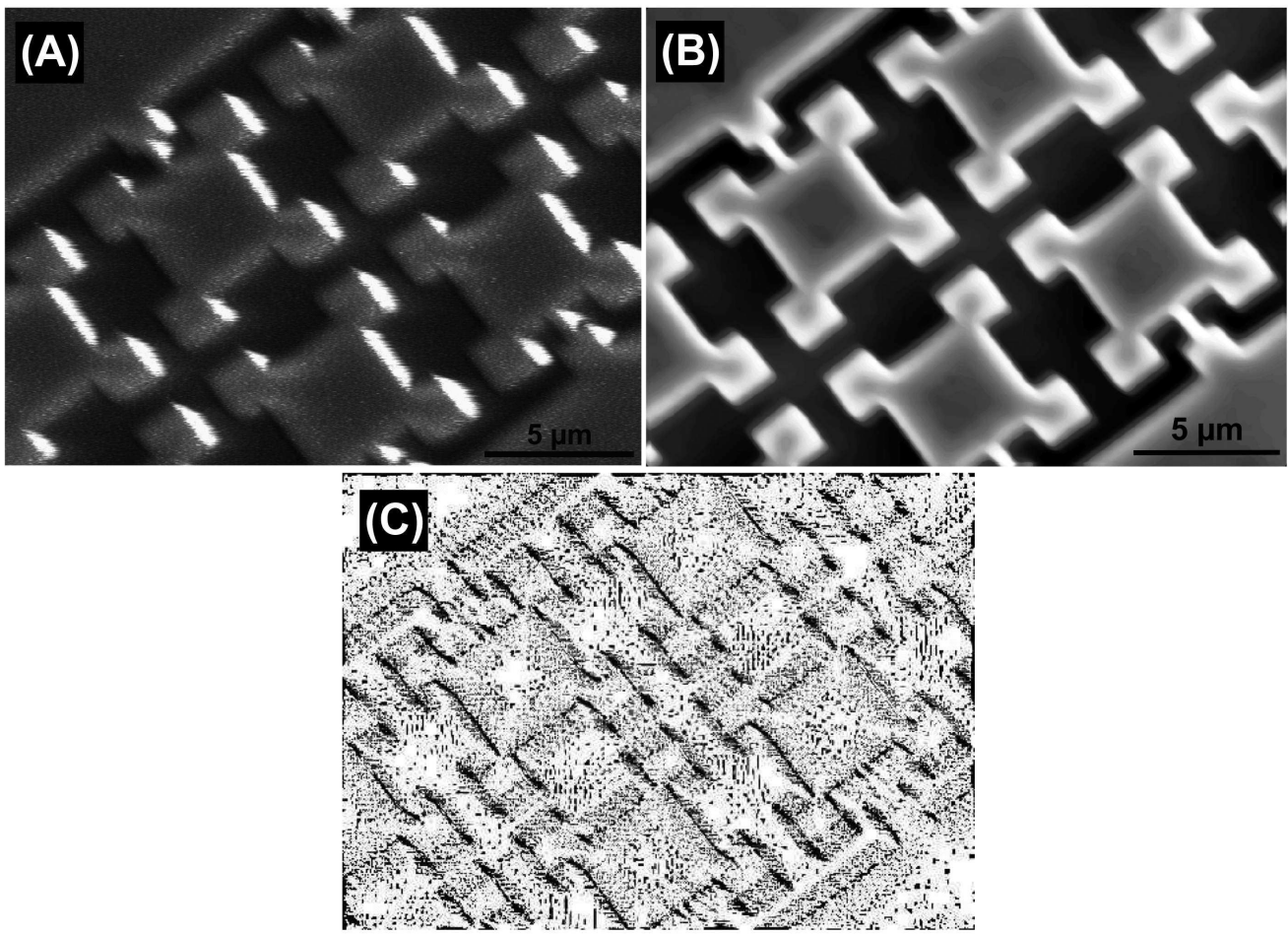

Figure 2 


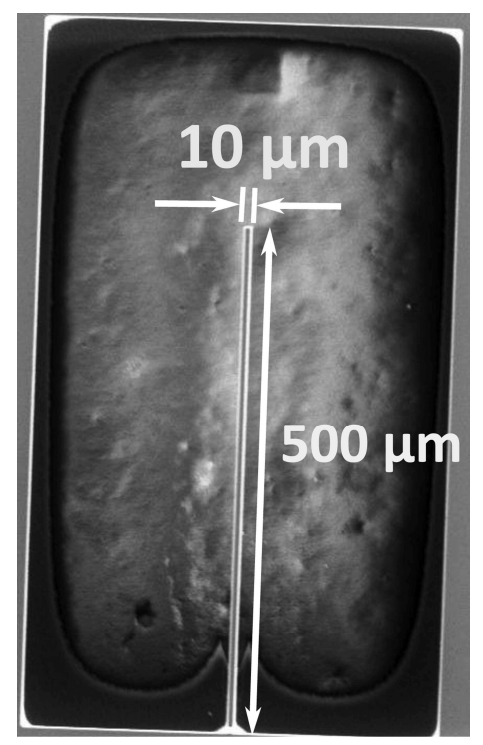

Figure 3
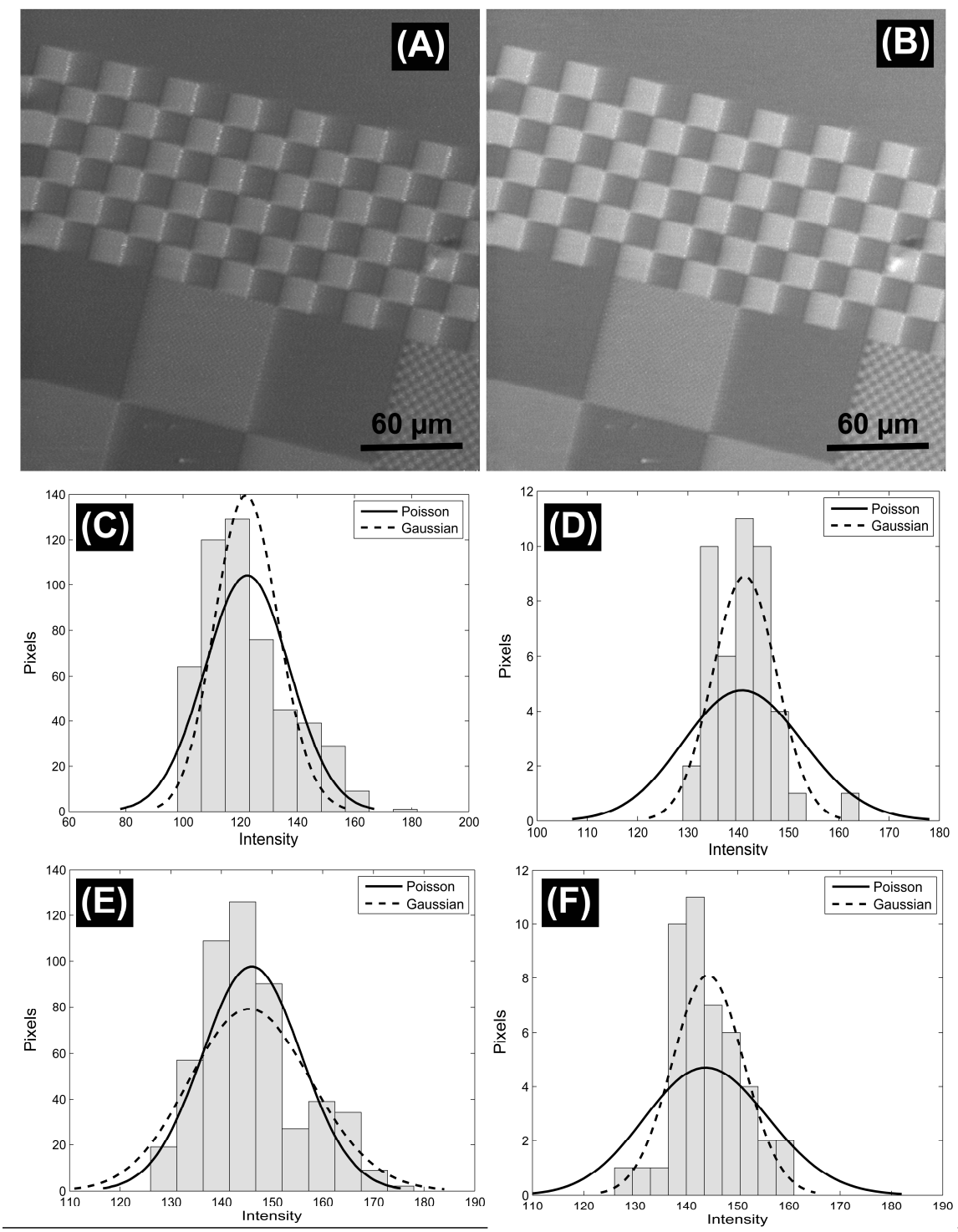

Figure 4 


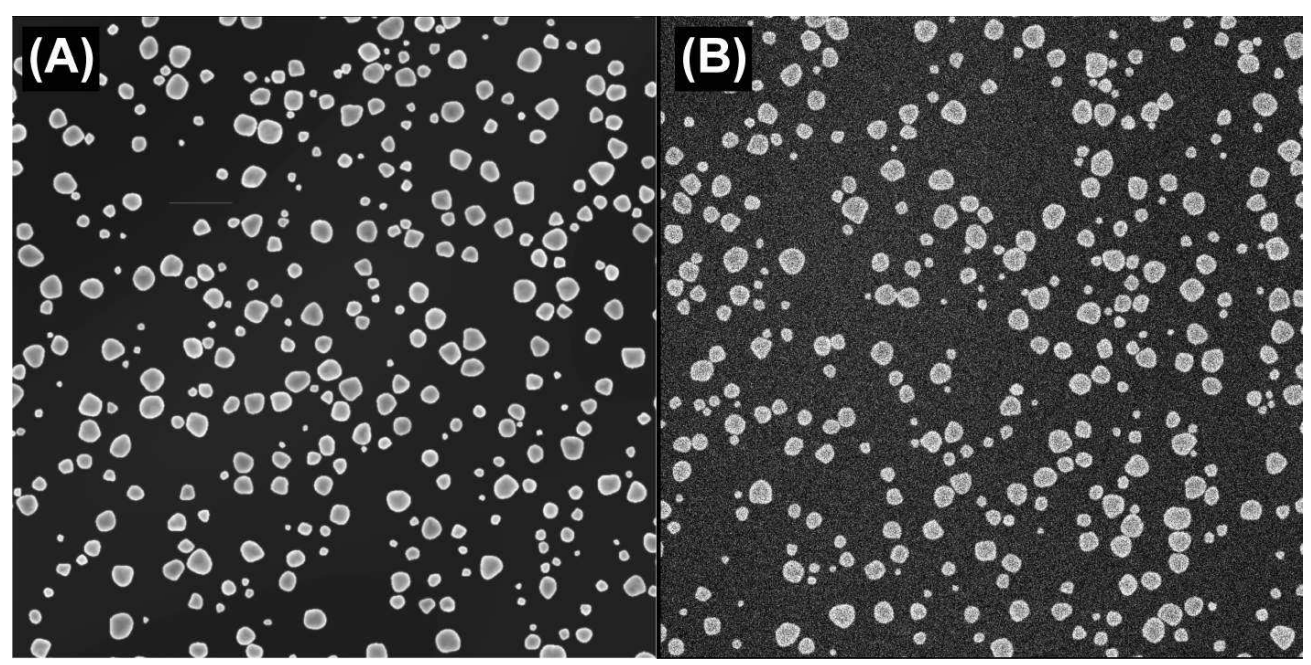

Figure 5
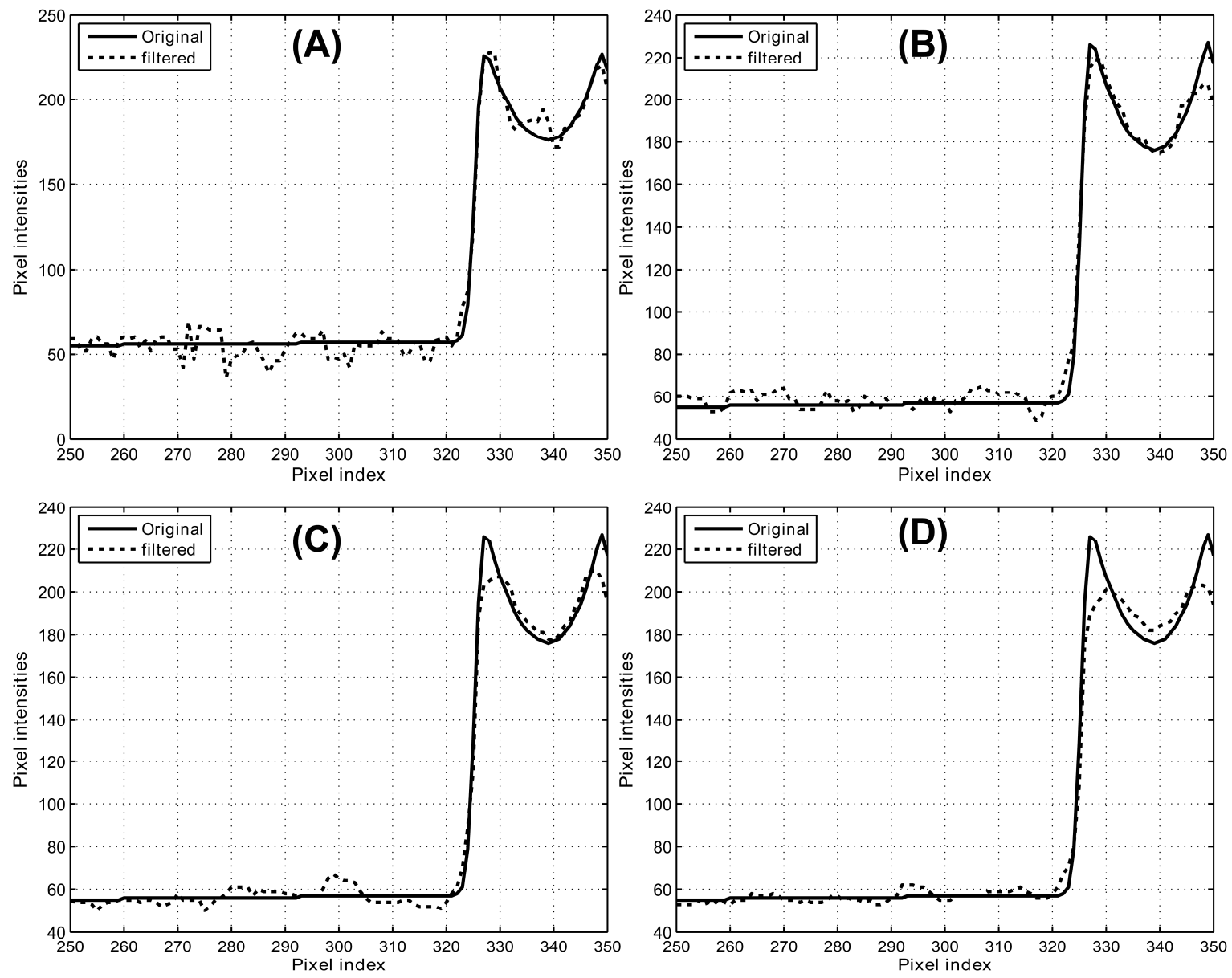

Figure 6 


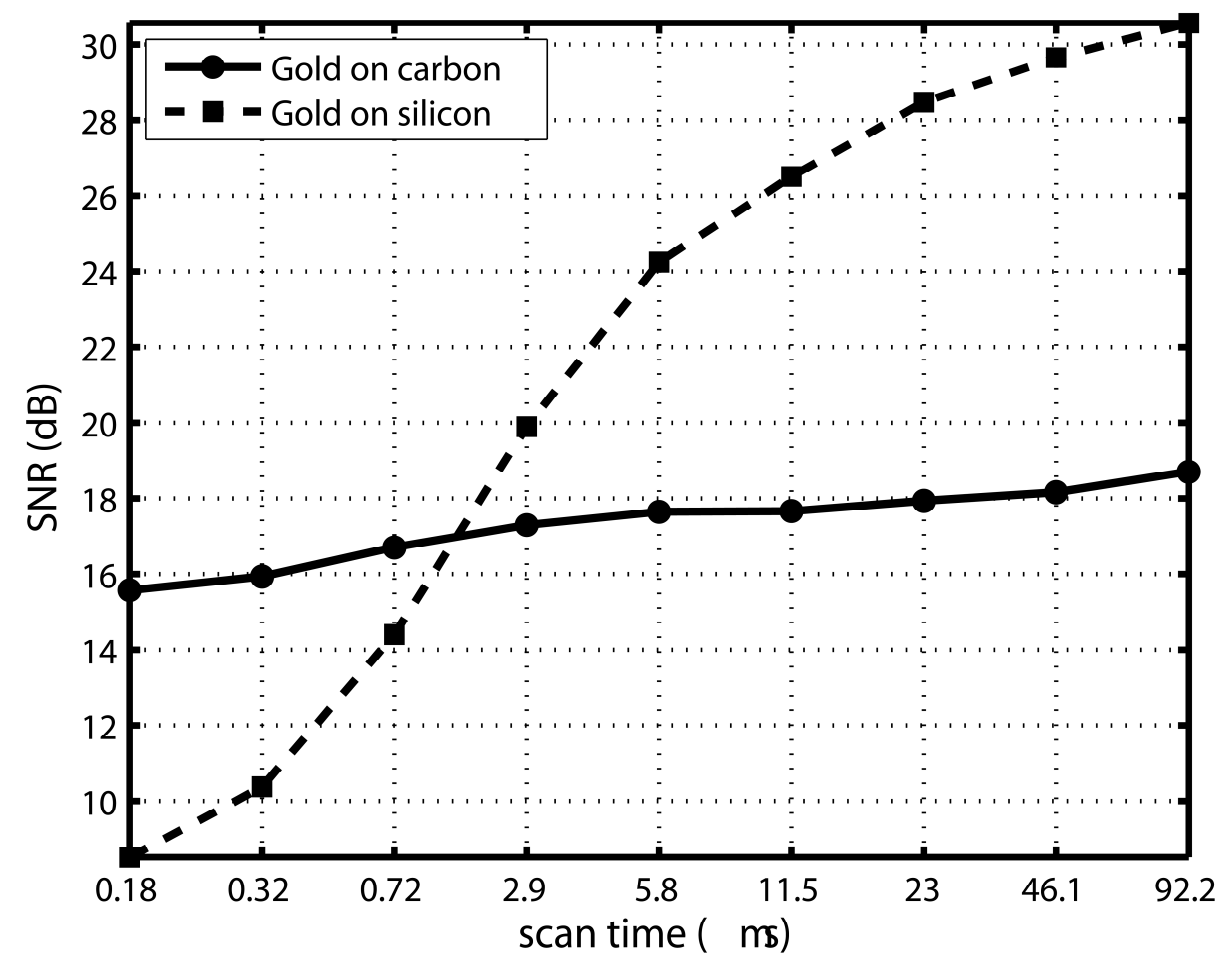

Figure 7

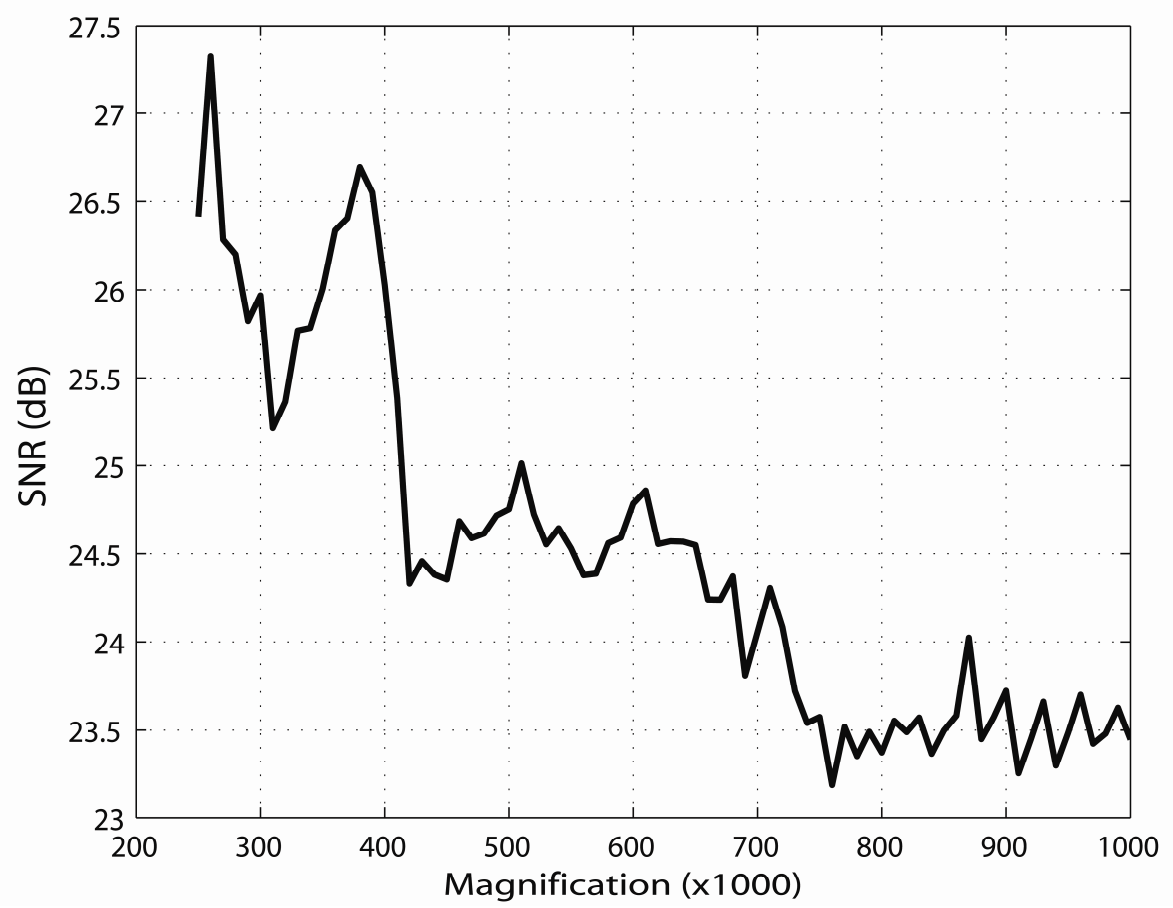

Figure 8 


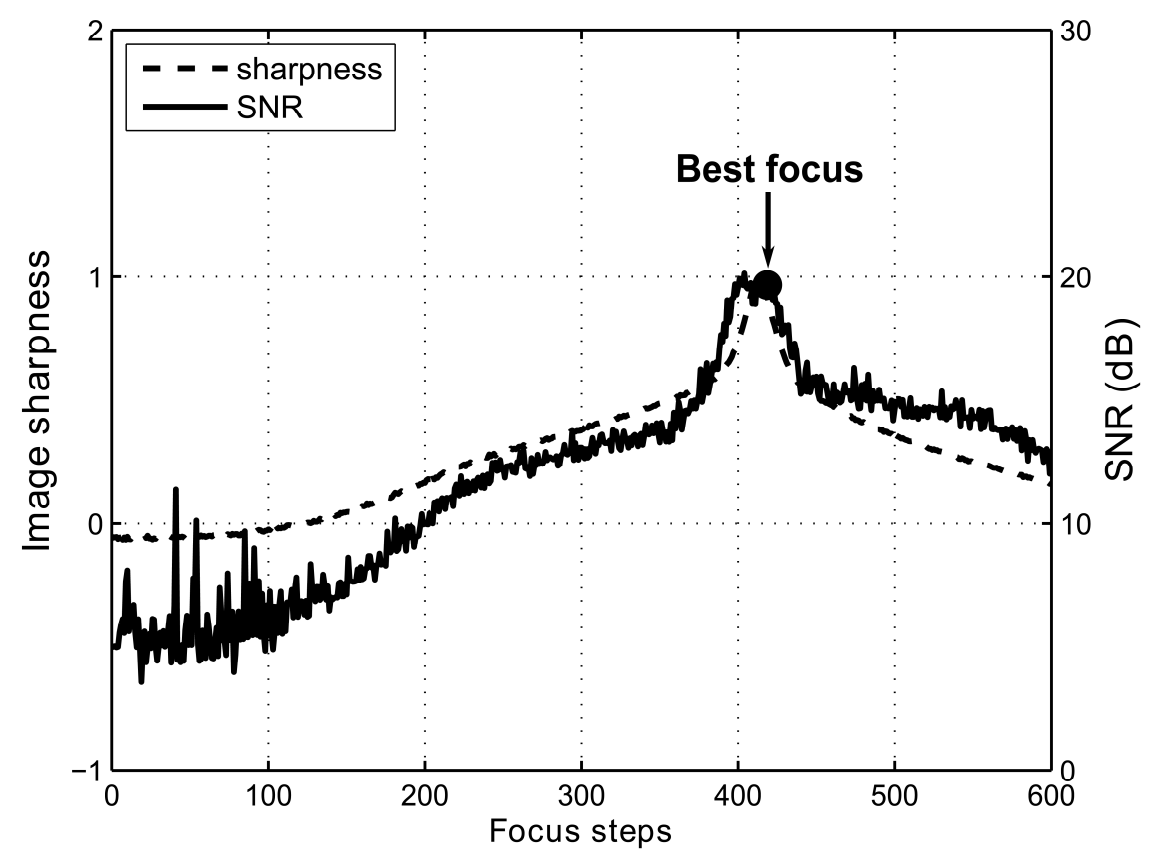

Figure 9

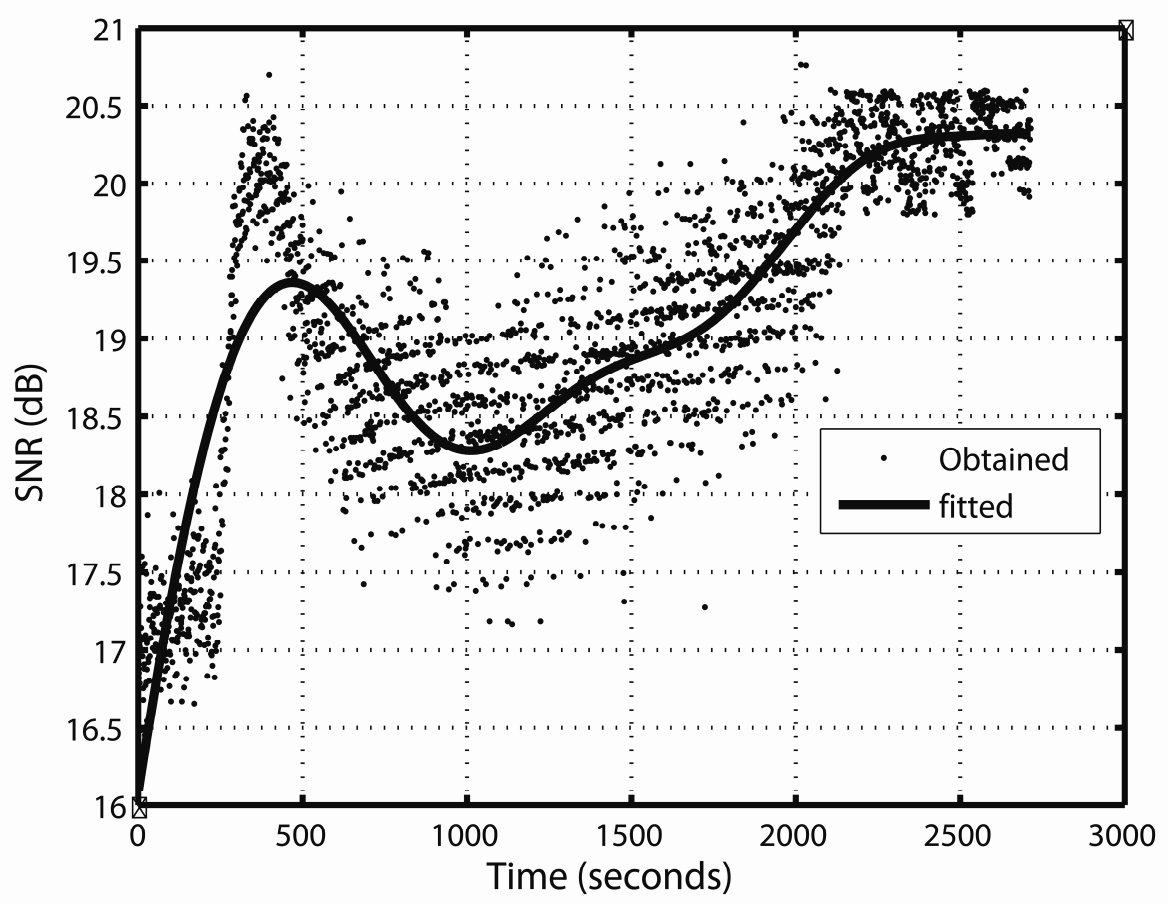

Figure 10 

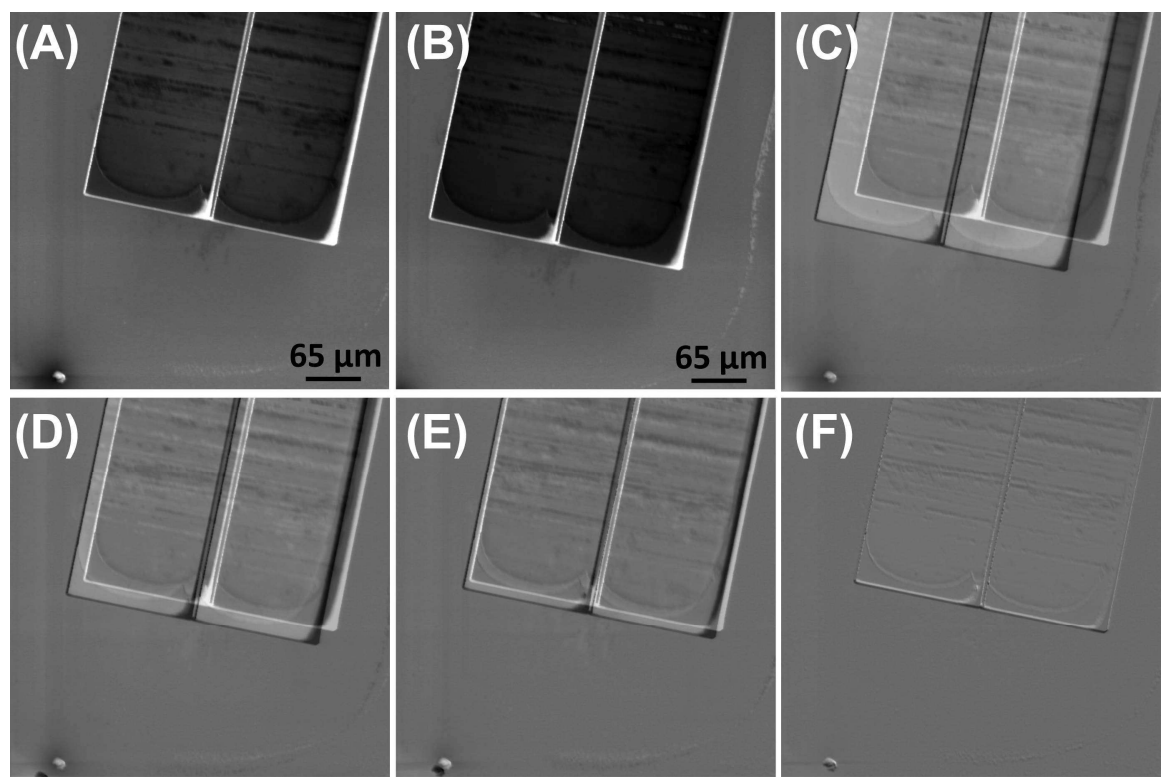

(F)

Figure 11

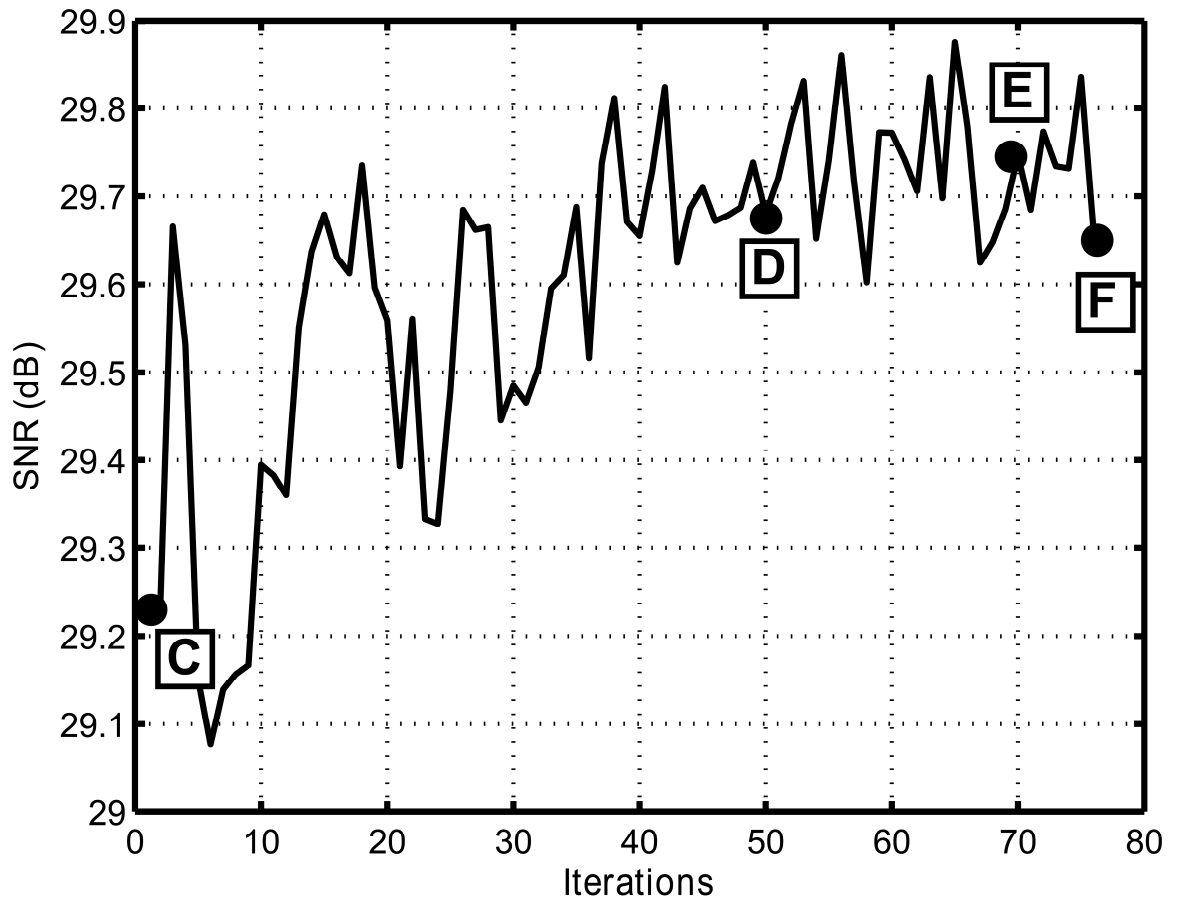

Figure 12 